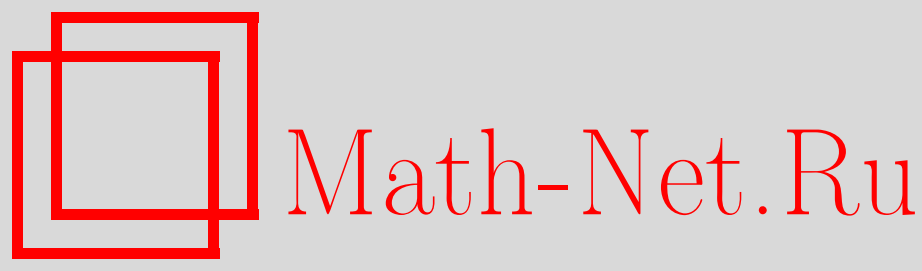

М. К. Волков, Г. М. Зиновьев, С. В. Молодцов, Мезонные корреляционные функции для модели с нелокальным четырехфермионным взаимодействием, ТМФ, 2009, том 161, номер 3, 406-419

DOI: https://doi.org/10.4213/tmf6450

Использование Общероссийского математического портала Math-Net.Ru подразумевает, что вы прочитали и согласны с пользовательским соглашением http://www . mathnet.ru/rus/agreement

Параметры загрузки:

IP : 54.166 .219 .16

26 апреля 2023 г., $17: 39: 11$

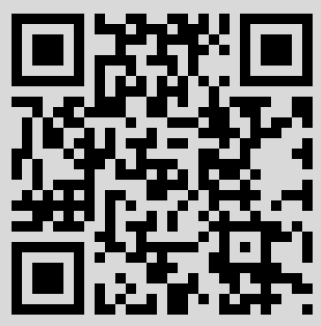




\title{
ФИЗИКА
}

Том 161, № 3

декабрь, 2009

\author{
2009 г. М. К. Волков*, Г. М. Зиновьев ${ }^{\dagger}$, С. В. Молодцов
}

\section{МЕЗОННЫЕ КОРРЕЛЯЦИОННЫЕ ФУНКЦИИ ДЛЯ МОДЕЛИ С НЕЛОКАЛЬНЫМ ЧЕТЫРЕХФЕРМИОННЫМ ВЗАИМОДЕЙСТВИЕМ}

\begin{abstract}
Изучаются мезонные корреляционные функции ряда моделей с четырехфермионным взаимодействием кварков. Показано, что несмотря на отмечавшийся ранее сингулярный характер средней энергии системы и кваркового конденсата, мезонные наблюдаемые конечны, вполне узнаваемы и сопоставимы по шкале энергий с данными эксперимента. Это позволяет использовать широкий набор гамильтонианов для моделирования неравновесных состояний кварковых и адронных систем, что является актуальной задачей при исследовании физики столкновений ультрарелятивистских тяжелых ионов. Для модели Келдыша получены аналитические выражения для мезонных корреляционных функций.
\end{abstract}

Ключевые слова: мезонная корреляционная функция, нелокальное четырехфермионное взаимодействие, модель Келдыша.

\section{1. ВВЕДЕНИЕ}

Изучение столкновений ультрарелятивистских тяжелых ионов выдвигает в качестве одной из важных задач описание неравновесных процессов в кварковой/адронной материи. Предшествуя термализации и установлению химического равновесия, эта стадия представляется на сегодняшний день сложной и, по-видимому, играет определяющую роль в процессе столкновения. Экспериментальные данные свидетельствуют, что в начальный момент соударения в системе проявляется большое число степеней свободы с сильным взаимодействием между составными частями системы. Например, характерное время неравновесной стадии на установке RHIC оценивается как 1 фм/с [1], [2]. Неожиданным представляется и отсутствие заметных сигналов о фазовых превращениях адронной материи, несмотря на зафиксированную в экспериментах высокую плотность энергии. Так, в установке RHIC она превышает 15 ГэВ $/$ фм $^{3}$, что заметно превосходит вакуумную плотность энергии (порядка нескольких сотен МэВ/фм $\left.{ }^{3}\right)$, а также плотность энергии барионной материи (константа мешка имеет порядок сотни МэВ $/$ рм$^{3}$ ). Зафиксировано короткое время

* Объединенный институт ядерных исследований, Дубна, Московская область, Россия

${ }^{\dagger}$ Институт теоретической физики им. Н. Н. Боголюбова НАНУ, Киев, Украина

${ }^{\ddagger}$ Институт теоретической и экспериментальной физики, Москва, Россия. E-mail: molodtsov@itep.ru 
неравновесной стадии, и это требует разработки схемы, пригодной для описания процессов формирования кварков с динамически генерированной массой.

Напомним, что описание равновесных состояний и фазовой диаграммы кварковой/адронной материи базируется в основном на модели Намбу-Иона-Лазинио (НИЛ) [3]. В рамках этой модели дается адекватная картина спонтанного нарушения киральной симметрии и успешно описывается низкоэнергетическая физика мезонов [4], [5]. Однако в применении к неравновесным процессам модель НИЛ непосредственно не применима. Например, необходимо описать связь двух групп кварков, с импульсами ниже и выше применяемого в модели параметра обрезания $\Lambda_{\mathrm{NJL}}$, а также включить глюонные степени свободы. Подобные затруднения приводят к необходимости поиска моделей, родственных модели НИЛ, которые содержат сопоставимое число эффективных степеней свободы в области низких энергий с более естественным описанием жесткой компоненты и, по возможности, являются столь же простыми, как и модель НИЛ. В работе [6] исследовались эффективные гамильтонианы с четырехфермионным взаимодействием в виде произведения двух разнесенных в пространстве кварковых токов, связанных посредством формфактора. Как и в модели НИЛ, полагалось, что основное состояние системы сформировано кварк-антикварковыми парами с противоположными импульсами. На основе приближения Хартри-Фока-Боголюбова удалось построить детальное описание кварков в виде квазичастиц, пригодное во всем диапазоне импульсов. Сопоставление моделей с дельтаобразным формфактором в координатном пространстве (модель НИЛ) и с дельтаобразным формфактором в импульсном пространстве (модель, родственная широко используемой в физике конденсированного состояния модели Келдыша [7]) показало, что даже эти, в определенном смысле взаимно противоположные, модели приводят при сопоставимых динамических массах кварков к эквивалентным квазичастицам.

В целом оказалось, что характеризующие квазичастицы параметры определяются некоторым динамическим механизмом, мало чувствительным к виду формфактора. Неожиданной особенностью рассмотренных моделей оказалась разрывность функционала средней энергии как функции токовой массы кварка. Как следствие, затруднительно фитировать кварковый конденсат вне кирального предела. Трактуемые буквально, киральный конденсат и средняя энергия кваркового ансамбля бесконечны. Однако указанные характеристики кваркового ансамбля не являются физическими наблюдаемыми, и, чтобы судить о пригодности моделей, следует обратиться, например, к мезонным корреляционным функциям. В настоящей работе подробно исследованы мезонные наблюдаемые в модели Келдыша. Можно сказать, что это наиболее простая, одномерная (в смысле, который будет пояснен ниже) модель мезонов. В целом ответ на вопрос о пригодности используемой модели оказывается положительным. Мезоны, несмотря на сингулярный характер вакуумного состояния, конечны, вполне узнаваемы, и их энергии соответствуют шкале энергий экспериментальных данных, хотя некоторые характеристики оказываются и не похожими на известные, что вполне естественно для такой простой модели. При динамической массе кварка, сопоставимой с моделью НИЛ, масса $\pi$-мезона для выбранных настроечных параметров оказывается несколько заниженной. Можно отметить также большое количество ветвей, определяющих зависимость энергии мезона от импульса, или, например, равенство нулю константы пионного распада $f_{\pi}$. 


\section{2. МОДЕЛЬНЫЙ ЛАГРАНЖКИАН}

Плотность модельного лагранжиана, изучавшегося в работе [6], имеет вид произведения двух цветных кварковых токов, которые локализованы в пространственных точках $\boldsymbol{x}$ и $\boldsymbol{y}$, связанных формфактором $F_{\mu \nu}$ :

$$
\mathcal{L}=\bar{q}\left(i \gamma_{\mu} \partial_{\mu}+i m\right) q-\bar{q} t^{a} \gamma_{\mu} q \int d \boldsymbol{y} G F_{\mu \nu}(\boldsymbol{x}-\boldsymbol{y}) \bar{q}^{\prime} t^{a} \gamma_{\nu} q^{\prime},
$$

где $q=q(\boldsymbol{x}, t), \bar{q}=\bar{q}(\boldsymbol{x}, t), q^{\prime}=q(\boldsymbol{y}, t), \bar{q}^{\prime}=\bar{q}(\boldsymbol{y}, t)-$ поля кварков, $t^{a}=\lambda^{a} / 2-$ генераторы цветовой калибровочной группы $S U\left(N_{\mathrm{c}}\right), m$ - токовая масса. Плотность лагранжиана приведена в евклидовом пространстве, $\gamma_{\mu}$ обозначают эрмитовы матрицы Дирака, $\mu, \nu=1,2,3,4$. Напомним, эффективный гамильтониан, соответствующий (1), появляется в результате использования процедуры усреднения при описании поведения кварков, находящихся под действием сильного стохастического глюонного поля. В качестве примера можно упомянуть (анти-)инстантонный ансамбль. В общем виде формфактор в выражении (1) может быть представлен в виде двух слагаемых: $F_{\mu \nu}(\boldsymbol{x}-\boldsymbol{y})=G F(\boldsymbol{x}-\boldsymbol{y}) \delta_{\mu \nu}+J_{\mu \nu}(\boldsymbol{x}-\boldsymbol{y})$, где подразумевается, что вторая компонента образована вектором относительного расстояния. В первом слагаемом выделена константа $G$, характеризующая силу четырехфермионного взаимодействия. В частном случае, когда формфактор $F$ имеет дельтаобразный вид в координатном пространстве, мы получаем модель НИЛ. Если же формфактор имеет дельтаобразный вид в импульсном пространстве, $F(\boldsymbol{p})=(2 \pi)^{3} \delta(\boldsymbol{p})$, то такая модель является аналогом широко используемой в физике конденсированного состояния модели Келдыша. Для простоты мы не будем рассматривать вклад коррелятора $J_{\mu \nu}(p)$. Проведенный в работе [6] подробный анализ системы вне кирального предела показал, что четырехкварковое взаимодействие сингулярно, поскольку средняя энергия ансамбля стремится к $-\infty$. Сингулярным также оказывается и кварковый

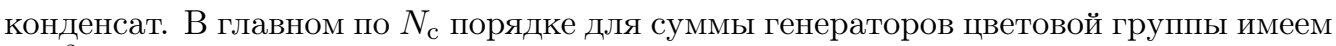
$\sum_{a=1}^{N_{\mathrm{c}}^{2}-1} t_{i j}^{a} t_{k l}^{a} \approx \delta_{i l} \delta_{k j} / 2$. Используя преобразование Фирца

$$
\gamma_{\mu} \otimes \gamma_{\mu}=1 \otimes 1+i \gamma_{5} \otimes i \gamma_{5}-\frac{1}{2} \gamma_{\mu} \otimes \gamma_{\mu}-\frac{1}{2} \gamma_{\mu} \gamma_{5} \otimes \gamma_{\mu} \gamma_{5}
$$

для скалярного вклада, в приближении среднего поля получим следующую эффективную лагранжеву плотность:

$$
\mathcal{L}=\bar{q}\left(i \gamma_{\mu} \partial_{\mu}+i m\right) q-\int d \boldsymbol{y} G F(\boldsymbol{x}-\boldsymbol{y})\left\langle\bar{q} q^{\prime}\right\rangle \bar{q}^{\prime} q
$$

(угловые скобки обозначают соответствующее среднее). Обратим также внимание, что член взаимодействия построен из бесцветных комбинаций кварковых операторов с перемешанными координатами точек $\boldsymbol{x}$ и $\boldsymbol{y}$. Условие самосогласованности приближения позволяет найти массу кварка:

$$
M(\boldsymbol{p})=2 N_{\mathrm{c}} \int \frac{d \boldsymbol{q}}{(2 \pi)^{3}} G F(\boldsymbol{p}-\boldsymbol{q}) \frac{m+M(\boldsymbol{q})}{\left[\boldsymbol{q}^{2}+(m+M(\boldsymbol{q}))^{2}\right]^{1 / 2}} .
$$

На этом этапе происходит спонтанное нарушение киральной симметрии. Для отвечающего модели НИЛ формфактора $F(\boldsymbol{x})=\delta(\boldsymbol{x})$ получаем хорошо известное 
уравнение для щели:

$$
M=2 N_{\mathrm{c}} G \int^{\Lambda_{\mathrm{NJL}}} \frac{d \boldsymbol{q}}{(2 \pi)^{3}} \frac{m+M}{\left[\boldsymbol{q}^{2}+(m+M)^{2}\right]^{1 / 2}},
$$

где $\Lambda_{\mathrm{NJL}}$ - параметр обрезания. Для модели Келдыша в приближении среднего поля имеем

$$
M(\boldsymbol{p})=2 N_{\mathrm{c}} G \frac{m+M(\boldsymbol{p})}{\left[\boldsymbol{p}^{2}+(m+M(\boldsymbol{p}))^{2}\right]^{1 / 2}} .
$$

На основании приведенного выражения легко заметить, что решение удобно представить как функцию $p(M)$. Нетрудно найти явную формулу для массы кварка в модели Келдыша в киральном пределе: $M(p)=\left[\left(2 N_{\mathrm{c}} G\right)^{2}-p^{2}\right]^{1 / 2}$. В дальнейшем анализе мы не станем строго придерживаться лагранжиана (1), а, руководствуясь правилом фирцевания, будем рассматривать более общее четырехфермионное взаимодействие: с константой $G$ для скалярного и псевдоскалярного каналов (поскольку киральная симметрия требует, чтобы константы в скалярном и псевдоскалярном каналах совпадали [3]) и константой $G_{\mathrm{V}}$ для векторного и аксиал-векторного каналов.

\section{3. БОЗОНИЗАЦИЯ}

В этом разделе мы введем мезонные поля, воспользовавшись хорошо известной процедурой бозонизации. Мы проведем рассмотрение на примере скалярного канала (аналогичные соотношения справедливы для псевдоскалярного, векторного и аксиал-векторного каналов). Удобно определить вспомогательные переменные

$$
\begin{aligned}
Q_{\mathrm{s}}(\boldsymbol{x}, \boldsymbol{y} ; t) & =F^{1 / 2}(\boldsymbol{x}-\boldsymbol{y}) \frac{1}{2}[\bar{q}(\boldsymbol{x} ; t) q(\boldsymbol{y} ; t)+\bar{q}(\boldsymbol{y} ; t) q(\boldsymbol{x} ; t)], \\
Q_{\mathrm{a}}(\boldsymbol{x}, \boldsymbol{y} ; t) & =F^{1 / 2}(\boldsymbol{x}-\boldsymbol{y}) \frac{1}{2}[\bar{q}(\boldsymbol{x} ; t) q(\boldsymbol{y} ; t)-\bar{q}(\boldsymbol{y} ; t) q(\boldsymbol{x} ; t)]
\end{aligned}
$$

для симметричной и антисимметричной комбинаций кварков. Поскольку формфактор является симметричной функцией по отношению к перестановке координат $\boldsymbol{x} \leftrightarrow \boldsymbol{y}$, интересующий нас вклад взаимодействия в скалярном секторе можно представить как

$\mathcal{V}_{\mathrm{int}}^{\mathrm{S}}=\frac{G}{2} F(\boldsymbol{x}-\boldsymbol{y}) \frac{1}{2}[\bar{q}(\boldsymbol{x} ; t) q(\boldsymbol{y} ; t) \bar{q}(\boldsymbol{y} ; t) q(\boldsymbol{x} ; t)+\bar{q}(\boldsymbol{y} ; t) q(\boldsymbol{x} ; t) \bar{q}(\boldsymbol{x} ; t) q(\boldsymbol{y} ; t)]=\frac{G}{2}\left(Q_{\mathrm{s}}^{2}-Q_{\mathrm{a}}^{2}\right)$.

Нетрудно видеть, что теперь применима стандартная процедура бозонизации, основанная на вспомогательном гауссовом интегрировании, с полями мезонов $\sigma_{\mathrm{s}}(\boldsymbol{x}, \boldsymbol{y} ; t)$ и $\sigma_{\mathrm{a}}(\boldsymbol{x}, \boldsymbol{y} ; t)$. Конкретно, интегрирование проводится с комбинациями

$$
\frac{\sigma_{\mathrm{s}}}{(2 G)^{1 / 2}}-\left(\frac{G}{2}\right)^{1 / 2} Q_{\mathrm{s}}, \quad \frac{i \sigma_{\mathrm{a}}}{(2 G)^{1 / 2}}+\left(\frac{G}{2}\right)^{1 / 2} Q_{\mathrm{a}}
$$

содержащими мезонные и кварковые поля. В результате взаимодействие представляется в виде, включающем мезонные поля:

$$
\begin{aligned}
\mathcal{V}_{\mathrm{int}}^{\mathrm{S}}= & \frac{G}{2}\left(Q_{\mathrm{s}}^{2}-Q_{\mathrm{a}}^{2}\right) \rightarrow-\frac{\sigma_{\mathrm{s}}^{2}+\sigma_{\mathrm{a}}^{2}}{2 G}+\frac{\sigma_{\mathrm{s}}+i \sigma_{\mathrm{a}}}{2} F^{1 / 2}(\boldsymbol{x}-\boldsymbol{y}) \bar{q}(\boldsymbol{x} ; t) q(\boldsymbol{y} ; t)+ \\
+ & \frac{\sigma_{\mathrm{s}}-i \sigma_{\mathrm{a}}}{2} F^{1 / 2}(\boldsymbol{x}-\boldsymbol{y}) \bar{q}(\boldsymbol{y} ; t) q(\boldsymbol{x} ; t) .
\end{aligned}
$$


Интегрируя далее по кварковым полям, получаем эффективную теорию, описывающую только мезоны. Мы не приводим соответствующих выкладок подробно, поскольку по сравнению со стандартной процедурой формально происходит всего лишь удвоение числа мезонных полей. Мы также не будем останавливаться на шагах, касающихся вывода уравнений для динамической массы кварка, а также получения мезонных корреляторов. Напомним только, что из первой вариации эффективного действия

$$
\frac{\sigma_{\mathrm{s}}^{(1)}}{G}+\left\langle Q_{\mathrm{s}}\right\rangle=0, \quad \frac{\sigma_{\mathrm{a}}^{(1)}}{G}+i\left\langle Q_{\mathrm{a}}\right\rangle=0
$$

получается уравнение, из которого можно найти динамическую массу кварка. В частности, выражение для симметричного поля эквивалентно уравнению (3). В результате для индуцированной массы кварка получим ${ }^{1)}$

$$
\begin{gathered}
M(\boldsymbol{x})=F^{1 / 2}(\boldsymbol{x}) \sigma_{\mathrm{s}}^{(1)}(\boldsymbol{x}) \\
\sigma_{\mathrm{s}}^{(1)}(\boldsymbol{x}-\boldsymbol{y})=-\frac{G}{2} F^{1 / 2}(\boldsymbol{x}-\boldsymbol{y})\langle\sigma|\bar{q}(\boldsymbol{x}) q(\boldsymbol{y})+\bar{q}(\boldsymbol{y}) q(\boldsymbol{x})| \sigma\rangle .
\end{gathered}
$$

Поскольку интерес представляют вещественные решения, мы рассмотрим только тот случай, когда средний вклад антисимметричной комбинации кварков обращается в нуль: $\left\langle Q_{\mathrm{a}}\right\rangle=0$.

Для квадратичных членов эффективного мезонного лагранжиана в скалярном канале имеем

$$
\begin{aligned}
& -\frac{\sigma_{\mathrm{s}}^{2}+\sigma_{\mathrm{a}}^{2}}{2 G}+\frac{1}{2}\left(\frac{\sigma_{\mathrm{s}}+i \sigma_{\mathrm{a}}}{2} \bar{q}(\boldsymbol{x} ; t) F^{1 / 2}(\boldsymbol{x}-\boldsymbol{y}) q(\boldsymbol{y} ; t)+\frac{\sigma_{\mathrm{s}}-i \sigma_{\mathrm{a}}}{2} \bar{q}(\boldsymbol{y} ; t) F^{1 / 2}(\boldsymbol{x}-\boldsymbol{y}) q(\boldsymbol{x} ; t)\right) \times \\
& \times\left(\frac{\sigma_{\mathrm{s}}^{\prime}+i \sigma_{\mathrm{a}}^{\prime}}{2} \bar{q}\left(\boldsymbol{x}^{\prime} ; t^{\prime}\right) F^{1 / 2}\left(\boldsymbol{x}^{\prime}-\boldsymbol{y}^{\prime}\right) q\left(\boldsymbol{y}^{\prime} ; t^{\prime}\right)+\frac{\sigma_{\mathrm{s}}^{\prime}-i \sigma_{\mathrm{a}}^{\prime}}{2} \bar{q}\left(\boldsymbol{y}^{\prime} ; t^{\prime}\right) F^{1 / 2}\left(\boldsymbol{x}^{\prime}-\boldsymbol{y}^{\prime}\right) q\left(\boldsymbol{x}^{\prime} ; t^{\prime}\right)\right) .
\end{aligned}
$$

Здесь подразумевается, что мезонные поля со штрихом взяты с координатами $\boldsymbol{x}^{\prime}, \boldsymbol{y}^{\prime}$ и временем $t^{\prime}$. Проводя спаривания и используя при этом соответствующие функции Грина, в импульсном представлении получим (в обозначениях мы опускаем интегрирования по соответствующим внутренним переменным)

$$
\begin{aligned}
& \sigma_{\alpha}\left(\boldsymbol{p}, \boldsymbol{q} ; p_{4}\right) K_{\alpha, \beta}^{\sigma}\left(\boldsymbol{p}, \boldsymbol{q} ; \boldsymbol{p}^{\prime}, \boldsymbol{q}^{\prime} ; p_{4}\right) \sigma_{\beta}\left(\boldsymbol{p}^{\prime}, \boldsymbol{q}^{\prime} ; p_{4}\right)= \\
& =\frac{\sigma_{\mathrm{s}}\left(\boldsymbol{p}, \boldsymbol{q} ; p_{4}\right) \sigma_{\mathrm{s}}\left(-\boldsymbol{p},-\boldsymbol{q} ;-p_{4}\right)+\sigma_{\mathrm{a}}\left(\boldsymbol{p}, \boldsymbol{q} ; p_{4}\right) \sigma_{\mathrm{a}}\left(-\boldsymbol{p},-\boldsymbol{q} ;-p_{4}\right)}{2 G}- \\
& \quad-\frac{1}{2} \operatorname{Tr} S\left(\boldsymbol{k} ; k_{4}\right) S\left(\boldsymbol{k}-\boldsymbol{p}-\boldsymbol{q} ; k_{4}-p_{4}\right)\left[\frac{\sigma_{\mathrm{s}}+i \sigma_{\mathrm{a}}}{2} F^{1 / 2}(\boldsymbol{k}-\boldsymbol{p}) F^{1 / 2}\left(\boldsymbol{k}+\boldsymbol{q}^{\prime}\right) \frac{\sigma_{\mathrm{s}}^{\prime}+i \sigma_{\mathrm{a}}^{\prime}}{2}+\right. \\
& \quad+\frac{\sigma_{\mathrm{s}}+i \sigma_{\mathrm{a}}}{2} F^{1 / 2}(\boldsymbol{k}-\boldsymbol{p}) F^{1 / 2}\left(\boldsymbol{k}+\boldsymbol{p}^{\prime}\right) \frac{\sigma_{\mathrm{s}}^{\prime}-i \sigma_{\mathrm{a}}^{\prime}}{2}+ \\
& \quad+\frac{\sigma_{\mathrm{s}}-i \sigma_{\mathrm{a}}}{2} F^{1 / 2}(\boldsymbol{k}-\boldsymbol{q}) F^{1 / 2}\left(\boldsymbol{k}+\boldsymbol{q}^{\prime}\right) \frac{\sigma_{\mathrm{s}}^{\prime}+i \sigma_{\mathrm{a}}^{\prime}}{2}+ \\
& \left.\quad+\frac{\sigma_{\mathrm{s}}-i \sigma_{\mathrm{a}}}{2} F^{1 / 2}(\boldsymbol{k}-\boldsymbol{q}) F^{1 / 2}\left(\boldsymbol{k}+\boldsymbol{p}^{\prime}\right) \frac{\sigma_{\mathrm{s}}^{\prime}-i \sigma_{\mathrm{a}}^{\prime}}{2}\right] .
\end{aligned}
$$

${ }^{1)}$ В импульсном представлении соответственно $M(\boldsymbol{p})=i(2 \pi)^{-3} G \int d \boldsymbol{q} F(\boldsymbol{p}-\boldsymbol{q}) \operatorname{Tr} S(\boldsymbol{q})$, где $S(\boldsymbol{q})-$ функция Грина для кварка. 
Здесь использованы обозначения $\sigma_{\mathrm{a}, \mathrm{s}}=\sigma_{\mathrm{a}, \mathrm{s}}\left(\boldsymbol{p}, \boldsymbol{q} ; p_{4}\right), \sigma_{\mathrm{a}, \mathrm{s}}^{\prime}=\sigma_{\mathrm{a}, \mathrm{s}}\left(\boldsymbol{p}^{\prime}, \boldsymbol{q}^{\prime} ;-p_{4}\right)$ и $\boldsymbol{q}^{\prime}=$ $-\boldsymbol{p}-\boldsymbol{q}-\boldsymbol{p}^{\prime}, \alpha, \beta=\mathrm{a}, \mathrm{s}$.

По-видимому, нет особой необходимости исследовать мезонные корреляционные функции в столь общей форме. Можно получить представление о виде решений, изучая некоторые частные случаи. Сначала рассмотрим условия, при которых формфакторы совпадают: $F^{1 / 2}(\boldsymbol{k}-\boldsymbol{q})=F^{1 / 2}\left(\boldsymbol{k}-\boldsymbol{p}-\boldsymbol{q}-\boldsymbol{p}^{\prime}\right), F^{1 / 2}(\boldsymbol{k}-\boldsymbol{q})=F^{1 / 2}\left(\boldsymbol{k}+\boldsymbol{p}^{\prime}\right)$. Из этих равенств видно, что импульсы кварков равны, $\boldsymbol{p}=\boldsymbol{q}$, т. е. в этой ситуации нет относительного движения кварков. Легко также заметить, что вклад антисимметричных полей в этом случае вырождается, а оставшаяся симметричная часть в точности соответствует стандартной процедуре бозонизации. В результате для мезонных корреляторов скалярного и псевдоскалярного каналов получим

$$
\begin{aligned}
K^{\sigma, \pi}=- & \frac{1}{2 G}+2 N_{\mathrm{c}} \int \frac{d k}{(2 \pi)^{4}} F(\boldsymbol{k}-\boldsymbol{p}) \times \\
& \times \frac{k_{4}\left(k_{4}-p_{4}\right)+\boldsymbol{k}(\boldsymbol{k}-2 \boldsymbol{p}) \mp(m+M(\boldsymbol{k}))(m+M(\boldsymbol{k}-2 \boldsymbol{p}))}{\left[k^{2}+(m+M(\boldsymbol{k}))^{2}\right]\left[\left(k_{4}-p_{4}\right)^{2}+(\boldsymbol{k}-2 \boldsymbol{p})^{2}+(m+M(\boldsymbol{k}-2 \boldsymbol{p}))^{2}\right]},
\end{aligned}
$$

где $k^{2}=k_{4}^{2}+\boldsymbol{k}^{2}$, знак "минус" отвечает $\sigma$-мезону, знак "плюс" $-\pi$-мезону. В частности, для модели Келдыша имеем

$$
K^{\sigma, \pi}=-\frac{1}{2 G}+2 N_{\mathrm{c}} \int \frac{d k_{4}}{2 \pi} \frac{k_{4}\left(k_{4}-p_{4}\right)-\boldsymbol{p}^{2} \mp(m+M(\boldsymbol{p}))^{2}}{\left[k_{4}^{2}+E^{2}(\boldsymbol{p})\right]\left[\left(k_{4}-p_{4}\right)^{2}+E^{2}(\boldsymbol{p})\right]},
$$

где используется обозначение $E^{2}(\boldsymbol{p})=\boldsymbol{p}^{2}+(m+M(\boldsymbol{p}))^{2}$ для энергии кварка. Для простоты записи формул будем опускать зависимость энергии $E$ от импульса. Существенным оказывается только интегрирование по компоненте $k_{4}$, и на основании этого можно сказать, что в данном случае мы получаем одномерную модель мезонов.

Знаменатель выражения (10) в корреляторе $\pi$-мезона удобно представить в следующем виде:

$$
\frac{1}{\left[k_{4}^{2}+E^{2}\right]\left[\left(k_{4}-p_{4}\right)^{2}+E^{2}\right]}=\frac{1}{\left[2 k_{4}\left(k_{4}-p_{4}\right)+p_{4}^{2}+2 E^{2}\right]}\left[\frac{1}{k_{4}^{2}+E^{2}}+\frac{1}{\left(k_{4}-p_{4}\right)^{2}+E^{2}}\right] .
$$

Теперь корреляционная функция $\pi$-мезона в (10) выражается через два интеграла,

$$
\begin{aligned}
K^{\pi}=- & \frac{1}{2 G}+2 N_{\mathrm{c}} \int \frac{d k_{4}}{2 \pi} \frac{1}{2}\left(\frac{1}{k_{4}^{2}+E^{2}}+\frac{1}{\left(k_{4}-p_{4}\right)^{2}+E^{2}}\right)- \\
& -N_{\mathrm{c}} \int \frac{d k_{4}}{2 \pi} \frac{p_{4}^{2}+4 p^{2}}{\left[k_{4}^{2}+E^{2}\right]\left[\left(k_{4}-p_{4}\right)^{2}+E^{2}\right]},
\end{aligned}
$$

вычисляя которые, окончательно получим

$$
K^{\pi}=-\frac{1}{2 G}+\frac{N_{\mathrm{c}}}{2 E}+N_{\mathrm{c}} \begin{cases}-\frac{p_{4}^{2}+4 p^{2}}{2 p_{4} E\left(p_{4}+2 i E\right)}, & \operatorname{Im} p_{4}>i E, \\ \frac{1}{2 E}-\frac{p_{4}^{2}+4 p^{2}}{E\left(p_{4}^{2}+4 E^{2}\right)}, & \left|\operatorname{Im} p_{4}\right|<E, \\ -\frac{p_{4}^{2}+4 p^{2}}{2 p_{4} E\left(p_{4}-2 i E\right)}, & \operatorname{Im} p_{4}<-i E,\end{cases}
$$




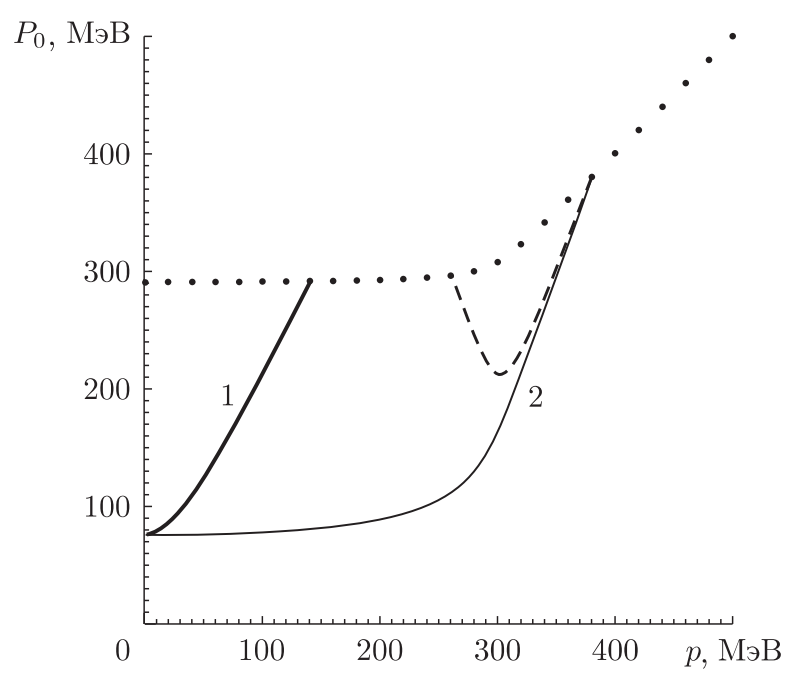

Рис. 1. Энергии $\sigma$-мезона (штриховая линия) и $\pi$-мезона (сплошные линии) как функции импульса. Жирная сплошная линия отвечает случаю нулевого относительного импульса кварков, тонкая сплошная линия описывает кварк-антикварковую систему с нулевым полным импульсом. Пунктиром обозначена энергия кварка $E$.

где $p=|\boldsymbol{p}|$. В евклидовой области для действительных значений энергии $p_{4}$ имеем

$$
K^{\pi}=-\frac{N_{\mathrm{c}}}{E\left(p_{4}^{2}+4 E^{2}\right)}\left(\frac{E}{\widetilde{G}} p_{4}^{2}+\frac{E-\widetilde{G}}{\widetilde{G}} 4 E^{2}+4 p^{2}\right),
$$

где $\widetilde{G}=2 N_{\mathrm{c}} G$. При этом мезонная корреляционная функция выглядит как некий фактор экранирования. Чтобы исследовать псевдоевклидов случай, будем считать, что переменная $p_{4}$ является чисто мнимой. Вводя обозначение $p_{4}=i P_{0}$, получим

$$
K^{\pi}=N_{\mathrm{c}} \begin{cases}\frac{1}{E\left(P_{0}^{2}-4 E^{2}\right)}\left(-\frac{E}{\widetilde{G}} P_{0}^{2}+\frac{E-\widetilde{G}}{\widetilde{G}} 4 E^{2}+4 p^{2}\right), & P_{0}<E, \\ \frac{1}{P_{0} E\left(P_{0}+2 E\right)}\left(-\frac{E}{\widetilde{G}} P_{0}^{2}+\frac{\widetilde{G}-2 E}{\widetilde{G}} E P_{0}+2 p^{2}\right), & P_{0}>E .\end{cases}
$$

Сравнивая полученное выражение при $P_{0}<E$ с формулой (12), видим, что переход от евклидовых переменных $p_{4}$ к псевдоевклидову случаю не изменяет формы выражения. В силу соображений симметрии мы не будем рассматривать ветвь $\operatorname{Im} p_{4}<-E$.

Найдем теперь закон дисперсии $\pi$-мезона, который определяется нулями корреляционной функции $K^{\pi}$. Результаты для скалярного и псевдоскалярного мезонов представлены на рис. 1, а для векторного и аксиал-векторного мезонов - на рис. 2 . Для ветви $P_{0}<E$ дисперсия дается уравнением

$$
P_{0}^{2}=4(E-\widetilde{G}) E+\frac{\widetilde{G}}{E}(2 p)^{2} .
$$


Из уравнения (4) для энергии кварка можно получить

$$
E=\widetilde{G} \frac{m+M}{M}
$$

В частности, при нулевом импульсе кварка для индуцированной массы кварка имеем $M(0)=\widetilde{G}$. Откуда для энергии $\pi$-мезона получим

$$
P_{0}^{2}=4(E-\widetilde{G}) E=4 m(m+\widetilde{G}),
$$

т. е. в киральном пределе $m \rightarrow 0$ теорема Голдстоуна выполняется (см. рис. 1 , кривая 1). Если настраивать параметры так, как это предложено в работе [6], то модели НИЛ [4] отвечают следующие параметры: $m=\left(m_{u}+m_{d}\right) / 2=5$ МэВ, $\widetilde{G}=286$ МэВ. Для энергии $\pi$-мезона при этом получим $P_{0} \approx 76$ МэВ при нулевом импульсе кварка. Для сравнения, энергия кварка в области малых импульсов $E \approx m+M \approx 286$ МэВ. В случае $P_{0}>E$ интересующая нас ветвь имеет вид $P_{0}=N_{\mathrm{c}} G-E+\left[\left(E-N_{\mathrm{c}} G\right)^{2}+4 N_{\mathrm{c}} G p^{2} / E\right]^{1 / 2}$. Анализ показывает, что она не удовлетворяет условию $P_{0}>E$.

Теперь обратимся к скалярному каналу. Подынтегральное выражение в (10) удобно представить в виде

$$
\begin{aligned}
& \frac{k_{4}\left(k_{4}-p_{4}\right)-E^{2}}{\left[k_{4}^{2}+E^{2}\right]\left[\left(k_{4}-p_{4}\right)^{2}+E^{2}\right]}= \\
& \quad=\frac{1}{2}\left[\frac{1}{k_{4}^{2}+E^{2}}+\frac{1}{\left(k_{4}-p_{4}\right)^{2}+E^{2}}\right]-\frac{1}{2} \frac{p_{4}^{2}+4 E^{2}}{\left[k_{4}^{2}+E^{2}\right]\left[\left(k_{4}-p_{4}\right)^{2}+E^{2}\right]} .
\end{aligned}
$$

В результате для корреляционной функции $\sigma$-мезона имеем

$$
\begin{aligned}
K^{\sigma}=- & \frac{1}{2 G}+2 N_{\mathrm{c}} \int \frac{d k_{4}}{2 \pi} \frac{1}{2}\left(\frac{1}{k_{4}^{2}+E^{2}}+\frac{1}{\left(k_{4}-p_{4}\right)^{2}+E^{2}}\right)- \\
& -N_{\mathrm{c}} \int \frac{d k_{4}}{2 \pi} \frac{p_{4}^{2}+4 E^{2}}{\left[k_{4}^{2}+E^{2}\right]\left[\left(k_{4}-p_{4}\right)^{2}+E^{2}\right]}
\end{aligned}
$$

Вычисляя интегралы, получим

$$
K^{\sigma}=-\frac{1}{2 G}+\frac{N_{\mathrm{c}}}{2 E}+N_{\mathrm{c}} \begin{cases}-\frac{p_{4}-2 i E}{2 p_{4} E}, & \operatorname{Im} p_{4}>i E, \\ -\frac{1}{2 E}, & \left|\operatorname{Im} p_{4}\right|<E, \\ -\frac{p_{4}+2 i E}{2 p_{4} E}, & \operatorname{Im} p_{4}<-i E .\end{cases}
$$

В псевдоевклидовом случае для ветви $P_{0}<E$ корреляционная функция $\sigma$-мезона вырожденна: $K^{\sigma}=-1 / 2 G$. Для $P_{0}>E$ имеем $K^{\sigma}=\left(-P_{0}+\widetilde{G}\right) / 2 G P_{0}$. Сравнивая с энергией кварка (14), видим, что при $P_{0}>E$ интересующих нас нулей у $K^{\sigma}$ нет.

Мы исследовали случай, когда относительный импульс кварка и антикварка равен нулю. Обратимся теперь к описанию кварк-антикварковой системы с нулевым суммарным импульсом: $\boldsymbol{p}+\boldsymbol{q}=\mathbf{0}$ (см. формулу (9)). Для импульсов кварков на 
выходе при этом возможны два варианта: $\boldsymbol{p}^{\prime}=\boldsymbol{p}$ или $\boldsymbol{p}^{\prime}=-\boldsymbol{p}$. В первом случае для корреляционных функций псевдоскалярного и скалярного каналов получаем

$$
\begin{aligned}
& \pi_{\alpha} K_{\alpha, \beta}^{\pi} \pi_{\beta}=-\frac{1}{2} \frac{\pi_{\mathrm{s}}^{2}+\pi_{\mathrm{a}}^{2}}{2 G}+2 N_{\mathrm{c}} \frac{\pi_{\mathrm{s}}^{2}-\pi_{\mathrm{a}}^{2}}{2} \int \frac{d k_{4}}{2 \pi} \frac{k_{4}\left(k_{4}-p_{4}\right)+p^{2}+(m+M)^{2}}{\left[k_{4}^{2}+E^{2}\right]\left[\left(k_{4}-p_{4}\right)^{2}+E^{2}\right]}, \\
& \sigma_{\alpha} K_{\alpha, \beta}^{\sigma} \sigma_{\beta}=-\frac{1}{2} \frac{\sigma_{\mathrm{s}}^{2}+\sigma_{\mathrm{a}}^{2}}{2 G}+2 N_{\mathrm{c}} \frac{\sigma_{\mathrm{s}}^{2}-\sigma_{\mathrm{a}}^{2}}{2} \int \frac{d k_{4}}{2 \pi} \frac{k_{4}\left(k_{4}-p_{4}\right)+p^{2}-(m+M)^{2}}{\left[k_{4}^{2}+E^{2}\right]\left[\left(k_{4}-p_{4}\right)^{2}+E^{2}\right]},
\end{aligned}
$$

где $\alpha, \beta=\mathrm{a}, \mathrm{s} . \mathrm{B}$ результате для $\pi$-мезона имеем

$$
K_{\mathrm{s}}^{\pi}=\left\{\begin{array}{ll}
\frac{-P_{0}+\widetilde{G}-2 E}{2 G\left(P_{0}+2 E\right)}, & P_{0}>E, \\
\frac{P_{4}^{2}-4 E(E-\widetilde{G})}{2 G\left(4 E^{2}-P_{0}^{2}\right)}, & P_{0}<E ;
\end{array} \quad K_{\mathrm{a}}^{\pi}= \begin{cases}\frac{-P_{0}-\widetilde{G}-2 E}{2 G\left(P_{0}+2 E\right)}, & P_{0}>E, \\
\frac{P_{4}^{2}-4 E(E+\widetilde{G})}{2 G\left(4 E^{2}-P_{0}^{2}\right)}, & P_{0}<E .\end{cases}\right.
$$

Закон дисперсии для корреляционной функции $K_{\mathrm{s}}^{\pi}$ в случае $P_{0}<E$ определяется уравнением

$$
P_{0}^{2}=4(E-\widetilde{G}) E
$$

согласующимся с формулой (13), в которой следует положить равным нулю полный импульс кварк-антикварковой пары $(2 p \rightarrow 0$, см. рис. 1 , кривая 2$)$. В случае корреляционной функции $K_{\mathrm{a}}^{\pi}$ нет решения, удовлетворяющего критерию $P_{0}<E$. Аналогичным образом при $P_{0}>E$ нет решений как для корреляционной функции $K_{\mathrm{s}}^{\pi}$, так и для ветви $K_{\mathrm{a}}^{\pi}$.

Для скалярного канала можно получить следующие выражения:

$$
\begin{aligned}
& K_{\mathrm{s}}^{\sigma}= \begin{cases}\frac{-E P_{0}^{2}+E P_{0}(\widetilde{G}-2 E)+2 \widetilde{G}(m+M)^{2}}{2 G E P_{0}\left(P_{0}+2 E\right)}, & P_{0}>E, \\
\frac{E P_{0}^{2}-4 E^{2}(E-\widetilde{G})-4 \widetilde{G}(m+M)^{2}}{2 G E^{2}\left(4 E^{2}-P_{0}^{2}\right)}, & P_{0}<E ;\end{cases} \\
& K_{\mathrm{a}}^{\sigma}= \begin{cases}\frac{-E P_{0}^{2}-E P_{0}(\widetilde{G}+2 E)-2 \widetilde{G}(m+M)^{2}}{2 G E P_{0}\left(P_{0}+2 E\right)}, & P_{0}>E, \\
\frac{E P_{0}^{2}-4 E^{2}(E-\widetilde{G})+4 \widetilde{G}(m+M)^{2}}{2 G E^{2}\left(4 E^{2}-P_{0}^{2}\right)}, & P_{0}<E .\end{cases}
\end{aligned}
$$

Для ветви $K_{\sigma}^{s}$, которая отвечает случаю $P_{0}<E$, закон дисперсии определяется уравнением

$$
P_{0}^{2}=4(E-\widetilde{G}) E+4 \frac{\widetilde{G}}{E}(m+M)^{2}
$$

(см. штриховую кривую на рис. 1). Для корреляционной функции $K_{\mathrm{a}}^{\sigma}$ в этом случае подходящего решения дисперсионного уравнения нет, поскольку нарушается условие $P_{0}<E$. Анализ корней корреляционных функций в случае $P_{0}>E$ аналогичным образом показывает, что приемлемых решений также нет. Мы не будем усложнять далее наше рассмотрение и опустим обсуждение случая $\boldsymbol{p}^{\prime}=-\boldsymbol{p}$, поскольку уже приведенных данных достаточно, чтобы стало понятным, насколько обширным может 
оказаться анализ ветвей решений. Отметим только, что наличие связанного состояния даже для имеющих достаточно большие противоположные импульсы частиц не отвечает интуитивным ожиданиям.

При нахождении константы пионного распада требуется вычислить петлевой интеграл, аналогичный (10), в котором вершина, описывающая слабое взаимодействие кварков, не содержит формфактора $F^{1 / 2}(\boldsymbol{k}-\boldsymbol{p})$, характерного для мезонных полей. В результате для модели Келдыша с дельтаобразным коррелятором в импульсном пространстве интеграл с оставшейся слабой сингулярностью формально обращается в нуль, и, следовательно, константа пионного распада $f_{\pi}$ равна нулю.

Точно так же, как скалярный и псевдоскалярный каналы, можно рассмотреть случай векторных и аксиал-векторных мезонов, при этом в полученных выше выражениях следует сделать замены $\bar{q} q \rightarrow \bar{q} \gamma_{\mu} q, \sigma \rightarrow V_{\mu}, \bar{q} i \gamma_{5} q \rightarrow \bar{q} \gamma_{5} \gamma_{\mu} q, \pi \rightarrow A_{\mu}$. Однако мы опустим соответствующее исследование, и количественные результаты получим, обратившись к другому описанию мезонных корреляторов - с помощью уравнений для вершинных функций.

\section{4. ВЕРШИННЫЕ ФУНКЦИИ}

В предыдущем разделе было продемонстрировано, что гауссово интегрирование проводится с симметричными и антисимметричными комбинациями вспомогательных мезонных полей, причем антисимметричные поля входят с мнимой единицей. Понятно, что соответствующий анализ точки перевала в функциональном интеграле в комплексном пространстве может оказаться довольно сложным, и желательно рассмотреть альтернативные способы изучения мезонных наблюдаемых, контролируя кварковые степени свободы. С этой целью обратимся к описанию, которое дается вершинными функциями (уравнениями Бете-Солпитера). Найдем эффективное взаимодействие кварков, суммируя ряд выделенных диаграмм:

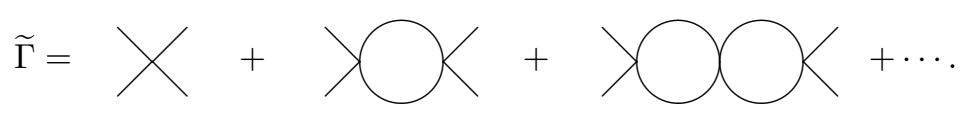

Следует принять во внимание, что, поскольку рассматриваются нелокальные мезонные поля, следовало бы изображать каждую линию удвоенной. Здесь первая диаграмма описывает затравочное взаимодействие $\Gamma_{0}(\boldsymbol{p}, \boldsymbol{q})=(2 \pi)^{3} G \Gamma F(\boldsymbol{p}-\boldsymbol{q})$, где матрица $Г$ обозначает канал взаимодействия: $1, i \gamma_{5}, \gamma_{\mu}, \gamma_{5} \gamma_{\mu}$. Выделяя при вершине комбинацию вида $\bar{q}_{\alpha}(\boldsymbol{x} ; t) \widetilde{\Gamma}_{\alpha \beta}(\boldsymbol{x}, \boldsymbol{y} ; t) q_{\beta}(\boldsymbol{y} ; t)$, для суммы ряда можно получить уравнение

$$
\begin{gathered}
-(2 \pi)^{3} G \Gamma F(\boldsymbol{p}-\boldsymbol{q})+\widetilde{\Gamma}\left(-\boldsymbol{p},-\boldsymbol{q} ; r_{4}\right)=-\int \frac{d k_{4} d \boldsymbol{k}}{2 \pi} G \Gamma F(-\boldsymbol{k}-\boldsymbol{q}) \operatorname{Tr} S\left(\boldsymbol{k} ; k_{4}\right) \times \\
\times \Gamma S\left(\boldsymbol{k}-\boldsymbol{p}+\boldsymbol{q} ; k_{4}-r_{4}\right) \widetilde{\Gamma}\left(\boldsymbol{k}-\boldsymbol{p}+\boldsymbol{q}, \boldsymbol{k} ; k_{4}-r_{4}\right),
\end{gathered}
$$

где $r_{4}=p_{4}-q_{4}$, а $p_{4}, q_{4}$ - четвертые компоненты импульсов кварка и антикварка. Решение для вершинной функции в модели Келдыша, например, ищем в виде 
$\widetilde{\Gamma}\left(\boldsymbol{p}, \boldsymbol{q} ; r_{4}\right)=(2 \pi)^{3} \Gamma \delta(\boldsymbol{p}-\boldsymbol{q}) V\left(\boldsymbol{p} ; r_{4}\right)$. Предполагается, что для мнимых значений $r_{4}$ в решении имеется особенность типа полюса. Выделяя сингулярные вклады, приближенно можно получить, что $-G+V=G \Pi V$, где поляризационный оператор в модели Келдыша представляется в виде

$$
\Pi=\int \frac{d k_{4}}{2 \pi} \operatorname{Tr} S\left(-\boldsymbol{p} ; k_{4}\right) \Gamma S\left(-\boldsymbol{p} ; k_{4}-r_{4}\right) \Gamma .
$$

Следовательно, для вершинной функции имеем $V=G /(1-G \Pi)$, и нули знаменателя определяют положение полюсов. В разделе, посвященном бозонизации, мы уже вычислили аналогичные поляризационные операторы. Опуская промежуточные выкладки, приведем выражения для псевдоскалярного и скалярного каналов при $\left|r_{4}\right|<E$ :

$$
\begin{aligned}
\frac{1-G \Pi^{\mathrm{PS}}}{\widetilde{G}} & =\frac{4 E(E-\widetilde{G})-R_{0}^{2}}{\widetilde{G}\left(4 E^{2}-R_{0}^{2}\right)}, \\
\frac{1-G \Pi^{\mathrm{S}}}{\widetilde{G}} & =\frac{\left(4 E^{2}-R_{0}^{2}\right) E+4\left[(m+M)^{2}-E^{2}\right] \widetilde{G}}{\widetilde{G} E\left(4 E^{2}-R_{0}^{2}\right)},
\end{aligned}
$$

где $R_{0}=P_{0}-Q_{0}, P_{0}=i p_{4}, Q_{0}=i q_{4}$. В псевдоскалярном случае закон дисперсии $\pi$-мезона совпадает с формулой (17), аналогично для $\sigma$-мезона получается выражение (18).

Для векторного и аксиал-векторного каналов в модели Келдыша получим (верхние знаки в формулах отвечают векторному мезону, нижние - аксиал-векторному мезону)

$$
\begin{aligned}
& \Pi_{44}^{\mathrm{V}, \mathrm{A}}=-4 N_{\mathrm{c}} \int \frac{d k_{4}}{2 \pi} \frac{k_{4}\left(k_{4}-r_{4}\right)-p^{2} \mp(m+M)^{2}}{\left[k_{4}^{2}+E^{2}\right]\left[\left(k_{4}-r_{4}\right)^{2}+E^{2}\right]}, \\
& \Pi_{4 i}^{\mathrm{V}, \mathrm{A}}=4 N_{\mathrm{c}} \int \frac{d k_{4}}{2 \pi} \frac{\left(2 k_{4}-r_{4}\right) p_{i}}{\left[k_{4}^{2}+E^{2}\right]\left[\left(k_{4}-r_{4}\right)^{2}+E^{2}\right]}, \\
& \Pi_{i j}^{\mathrm{V}, \mathrm{A}}=4 N_{\mathrm{c}} \int \frac{d k_{4}}{2 \pi} \frac{k_{4}\left(k_{4}-r_{4}\right) \delta_{i j}-2 p_{i} p_{j}+\delta_{i j} p^{2} \pm \delta_{i j}(m+M)^{2}}{\left[k_{4}^{2}+E^{2}\right]\left[\left(k_{4}-r_{4}\right)^{2}+E^{2}\right]},
\end{aligned}
$$

$\Pi_{4 i}^{\mathrm{V}, \mathrm{A}}=\Pi_{i 4}^{\mathrm{V}, \mathrm{A}}$. Легко видеть, что это выражение совпадает с соответствующей мезонной корреляционной функцией из предыдущего раздела. Вычислив интегралы в векторном и аксиал-векторном каналах, для случая $\operatorname{Im} r_{4}>E$ имеем

$$
\begin{aligned}
\Pi_{44}^{\mathrm{V}} & =-2 N_{\mathrm{c}} \frac{i}{r_{4}}, & \Pi_{44}^{\mathrm{A}} & =-\frac{2 N_{\mathrm{c}}}{E} \frac{i r_{4} E-2 p^{2}}{r_{4}\left(r_{4}+2 i E\right)}, \\
\Pi_{4 i}^{\mathrm{V}} & =-2 N_{\mathrm{c}} \frac{p_{i}}{r_{4} E}, & \Pi_{4 i}^{\mathrm{A}} & =\Pi_{4 i}^{\mathrm{V}}, \\
\Pi_{i j}^{\mathrm{V}} & =2 N_{\mathrm{c}} \frac{i r_{4} E \delta_{i j}-2 p_{i} p_{j}}{r_{4}\left(r_{4}+2 i E\right) E}, & \Pi_{i j}^{\mathrm{A}} & =\frac{2 N_{\mathrm{c}}}{E} \frac{\left[i r_{4} E-2(m+M)^{2}\right] \delta_{i j}-2 p_{i} p_{j}}{r_{4}\left(r_{4}+2 i E\right)} .
\end{aligned}
$$

В случае $\left|r_{4}\right|<E$ получим

$$
\begin{array}{ll}
\Pi_{44}^{\mathrm{V}}=0, & \Pi_{44}^{\mathrm{A}}=-\frac{8 N_{c}}{E} \\
\Pi_{4 i}^{\mathrm{V}}=0, & \Pi_{4 i}^{\mathrm{A}}=\Pi_{4 i}^{\mathrm{V}},
\end{array}
$$




$$
\Pi_{i j}^{\mathrm{V}}=8 N_{\mathrm{c}} \frac{E^{2} \delta_{i j}-p_{i} p_{j}}{E\left(r_{4}^{2}+4 E^{2}\right)}, \quad \Pi_{i j}^{\mathrm{A}}=\frac{8 N_{\mathrm{c}}}{E} \frac{p^{2} \delta_{i j}-p_{i} p_{j}}{r_{4}^{2}+4 E^{2}} .
$$

Теперь диагонализуем корреляционные функции, воспользовавшись тем обстоятельством, что соответствующие квадратичные формы определяют лагранжиан свободных векторных и аксиал-векторных мезонов:

$$
K^{\mathrm{V}, \mathrm{A}}=\widetilde{C}_{44}^{\mathrm{V}, \mathrm{A}} V_{4}^{2}+2 \widetilde{C}_{4 i}^{\mathrm{V}, \mathrm{A}} V_{4} \widetilde{V}_{i}+\widetilde{V}_{i} \widetilde{C}_{i j}^{\mathrm{V}, \mathrm{A}} \widetilde{V}_{j}
$$

(здесь подразумевается суммирование по повторяющимся индексам). По определению $\widetilde{C}_{\mu \nu}^{\mathrm{V}, \mathrm{A}}=\delta_{\mu \nu}-G_{\mathrm{V}} \Pi_{\mu \nu}^{\mathrm{V}, \mathrm{A}}$.

Переопределим пространственные компоненты (аксиал-)векторных полей с помощью замены $\widetilde{V}_{i}=V_{i}+\alpha p_{i} V_{4}$, где $\alpha^{\mathrm{V}, \mathrm{A}}=-\widetilde{C}_{4 i}^{\mathrm{V}, \mathrm{A}} p_{i} /\left(p_{i} \widetilde{C}_{i j}^{\mathrm{V}, \mathrm{A}} p_{j}\right)$ и уберем смешанные компоненты $\left(V_{4} V_{i}\right)$ в квадратичной форме $(23)$. В результате четвертая компонента векторного поля входит в квадратичную форму с коэффициентом $C_{44}^{\mathrm{V}, \mathrm{A}}=$ $\widetilde{C}_{44}^{\mathrm{V}, \mathrm{A}}-\left(\widetilde{C}_{4 i}^{\mathrm{V}, \mathrm{A}} p_{i}\right)^{2} /\left(p_{i} \widetilde{C}_{i j}^{\mathrm{V}, \mathrm{A}} p_{j}\right)$. Компоненты тензора $C_{i j}^{\mathrm{V}, \mathrm{A}}$ остаются прежними. Численный анализ показывает, что приемлемое решение для дисперсии четвертой компоненты имеется только для аксиал-векторного поля в случае $R_{0}>E$, однако мы не будем обсуждать это решение в настоящей работе. Пространственные составляющие векторных полей будем искать в двух формах - поперечной и продольной,

$$
V_{i}=\left(\delta_{i j}-\frac{p_{i} p_{j}}{p^{2}}\right) v_{j}^{\perp} \quad \text { и } \quad V_{i}=p_{i} v^{\|}
$$

соответственно.

Сначала рассмотрим случай $R_{0}>E$. Закон дисперсии поперечной компоненты векторного поля $v^{\perp}$ дается выражением $R_{0}=\widetilde{G}_{\mathrm{V}}-2 E$, где $\widetilde{G}_{\mathrm{V}}=2 N_{\mathrm{c}} G_{\mathrm{V}}$. Следовательно, нетривиальное решение возможно, если $\widetilde{G}_{\mathrm{V}}>3 E$. Для определенности положим, что $\widetilde{G}_{\mathrm{V}}=1.5 \widetilde{G}$, при малых импульсах кварков можно записать $\widetilde{G}_{\mathrm{V}} \approx 1.5 E$, так что для выбранной выше настройки параметров эта ветвь не проявляется. Дисперсия продольной составляющей $v^{\|}$в этом случае определяется как $R_{0}=N_{\mathrm{c}} G_{\mathrm{V}}-E+\left[\left(E-N_{\mathrm{c}} G_{\mathrm{V}}\right)^{2}+4 N_{\mathrm{c}} G_{\mathrm{V}} p^{2} / E\right]^{1 / 2}$.

Ветви мезонных наблюдаемых в векторном и аксиал-векторном каналах представлены на рис. 2. Рассмотренная выше ветвь изображена на рисунке сплошной линией, помеченной номером 1. Для поперечной составляющей аксиал-векторного мезона $a^{\perp}$ можно получить $R_{0}=N_{\mathrm{c}} G_{\mathrm{V}}-E+\left[\left(E-N_{\mathrm{c}} G_{\mathrm{V}}\right)^{2}+4 N_{\mathrm{c}} G_{\mathrm{V}}(m+M)^{2} / E\right]^{1 / 2}$. Эта кривая изображена на рис. 2 пунктирной линией с номером 2. Дисперсия продольной компоненты $a^{\|}$определяется как $R_{0}=\widetilde{G}_{\mathrm{V}}$. Эта ветвь появляется при $\widetilde{G}_{\mathrm{V}}>E$. На рис. 2 ей соответствует штриховая прямая с номером 3. При малых импульсах продольная $a^{\|}$и поперечная $a^{\perp}$ компоненты почти совпадают.

Теперь обратимся к случаю $R_{0}<E$. Дисперсия поперечной компоненты векторного мезона $v^{\perp}$ определяется как $R_{0}^{2}=4 E\left(E-\widetilde{G}_{\mathrm{V}}\right)$. Эта кривая изображена на рис. 2 сплошной кривой с номером 4. Для продольной составляющей $v^{\|}$имеем $R_{0}^{2}=$ $4\left(E^{2}-\widetilde{G}_{\mathrm{V}}(m+M)^{2} / E\right)$. Ей отвечает сплошная кривая с номером 5. Для поперечной компоненты аксиал-векторного мезона $a^{\perp}$ можно получить $R_{0}^{2}=4 E^{2}-8 N_{\mathrm{c}} G_{\mathrm{V}} p^{2} / E$. Эта кривая представлена штриховой линией с номером 6. Ее правая компонента почти совпадает с кривой 4 , поскольку при больших импульсах индуцированная 


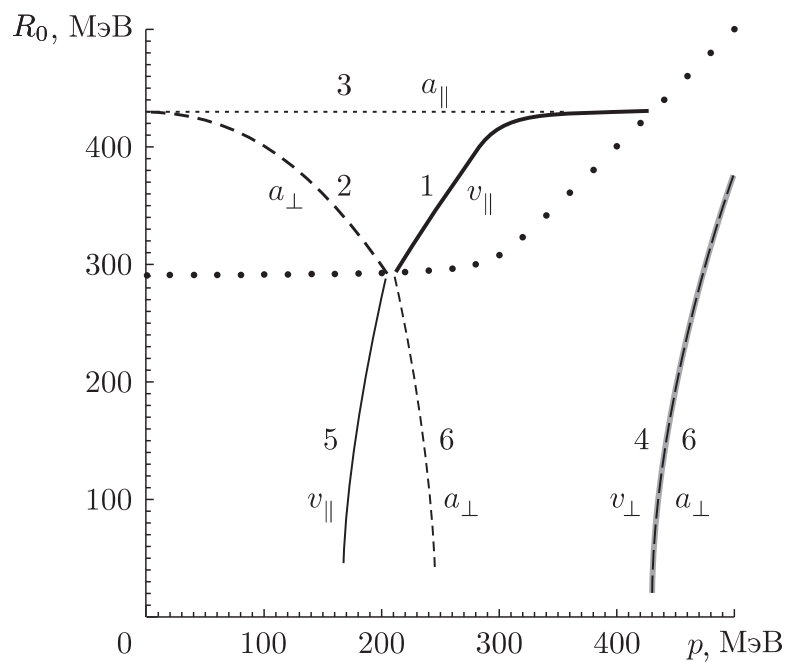

Рис. 2. Относительная энергия $R_{0}$ векторного (сплошные линии) и аксиал-векторного (штриховые линии) мезонов как функции импульса. Пояснения обозначений линий даны в тексте. Пунктиром обозначена энергия кварка $E$.

масса кварка стремится к нулю и происходит восстановление киральной симметрии. Продольная компонента аксиал-векторного поля $a^{\|}$вырожденна.

Напомним, для выбранных настроечных параметров энергия $\pi$-мезона в модели НИЛ совпадает с экспериментальными данными и имеет порядок $E_{\pi}^{\mathrm{NJL}}=140$ МэВ. Для модели Келдыша в работе [6] полагалось, что динамическая масса кварка в области малых импульсов совпадает с динамической массой модели НИЛ. Это условие приводит к почти полной идентичности квазичастиц обеих моделей. При этом энергия $\pi$-мезона в модели Келдыша получается несколько заниженной: $E_{\pi}^{\mathrm{K}}=76$ МэВ. Тем не менее интересно отметить, что выдержан относительный масштаб масс для разных каналов: аксиал-векторный мезон тяжелее векторного, а самым легким оказывается псевдоскалярное возбуждение. Можно провести и другое фитирование, используя наблюдаемые значения масс $\pi$-мезона, векторного мезона и аксиал-векторного мезона. Для $\pi$-мезона мы берем значение энергии, отвечающее нулевому значению импульса кварка, $M_{\pi}=2((m+\widetilde{G}) m)^{1 / 2}$. В случае векторных частиц используем три ветви для $R_{0}>E$, которым отвечают линии на рис. 2 , обозначенные как $1,2,3$, причем в случае аксиал-векторного мезона мы используем значение энергии при нулевом импульсе кварка, $M_{\mathrm{A}}=\widetilde{G}_{\mathrm{V}}$. Энергию векторного мезона (см. кривую 1 на рис. 2) положим равной энергии кварка, которая в этой области импульсов приближенно совпадает с динамической массой кварка и равна $M_{\mathrm{V}}=m+\widetilde{G}$. Используя экспериментальные данные $M_{\pi}=140 \mathrm{MэB}, M_{\mathrm{V}}=770 \mathrm{MэB}, M_{\mathrm{A}}=1260 \mathrm{MэB}$, для трех настроечных параметров получим $m \simeq 6.4 \mathrm{M \ni B,} \widetilde{G} \simeq 763 \mathrm{MэB}, \widetilde{G}_{\mathrm{V}} / \widetilde{G}=1.65$.

При вычислении корреляционных функций мы считали контур интегрирования фиксированным и не использовали сдвиг переменной интегрирования для придания интегралам симметричной формы. В силу одномерности задачи имеется оче- 
видная возможность также изучить зависимость корреляционных функций от вида контура. В частности, интерес представляет разворот контура интегрирования на мнимую ось. При этом два кварковых полюса можно будет трактовать также как вигнеровскую фазу и строить продолжения корреляционных функций, отличные от рассмотренных в работе. Это, однако, далеко выходит за рамки сформулированных в начале статьи целей исследования.

\section{5. ВЫВОДЫ}

Итак, несмотря на отмеченную в работе [6] сингулярность функционала средней энергии и кваркового конденсата, мезонные наблюдаемые конечны и хорошо согласуются со значениями, получаемыми в эксперименте. При этом эффективное число степеней свободы, задействованных в формировании квазичастиц, в модели НИЛ и в модели Келдыша приблизительно одинаково. Обладая одновременно простотой модели НИЛ, модель Келдыша представляется вполне пригодным кандидатом для описания неравновесных состояний кварк-антикваркового ансамбля. В силу одномерности модели Келдыша несложно описать аналитическое продолжение мезонных корреляционных функций из евклидова пространства в псевдоевклидово. Интересно также отметить, что связанные состояния обнаруживаются даже при достаточно больших противоположных импульсах кварков. Главный же вывод состоит в том, что для различных моделей при сопоставимых квазичастицах получаются сопоставимые мезонные корреляционные функции.

\section{Список литературы}

[1] А.Г. Литвиненко, ЭЧАЯ, 38:2 (2007), 409-459; P. Levai, "Could we find quark-gluon plasma in pp collision at LHC?", Selected Problems of Modern Theoretical Physics, XIII Int. Conf. (Dubna, June 23-27, 2008).

[2] B. Back et al. (PHOBOS Collab.), Nucl. Phys. A, 757:1-2 (2005), 28-101; J. Adams et al. (STAR Collab.), 102-183; K. Adcox et al. (PHENIX Collab.), 184-283.

[3] Y. Nambu, G. Jona-Lasinio, Phys. Rev., 122:1 (1961), 345-358.

[4] М. К. Волков, Д. Эберт, ЯФ, 36:5(11) (1982), 1265-1277; D. Ebert, M. K. Volkov, Z. Phys. C, 16:3 (1983), 205-210; M. K. Volkov, Ann. Phys., 157:1 (1984), 282-303; М. К. Волков, ЭЧАЯ, 17:3 (1986), 433-471; 24:1 (1993), 81-139; D. Ebert, H. Reinhardt, M. K. Volkov, Progr. Part. Nucl. Phys., 33 (1994), 1-120; D. Ebert, H. Reinhardt, Nucl. Phys. B, 271:3-4 (1986), 188-226.

[5] М. К. Волков, А. Е. Раджабов, УФН, 176:6 (2006), 569-580; S. P. Klevansky, Rev. Modern Phys., 64:3 (1992), 649-708; U. Vogl, W. Weise, Progr. Part. Nucl. Phys., 27 (1991), 195-272; T. Hatsuda, T. Kunihiro, Phys. Rep., 247:5-6 (1994), 221-367.

[6] Г. М. Зиновьев, С.В. Молодцов, ТМФ, 160:3 (2009), 444-470; S. V. Molodtsov, G. M. Zinovjev, Phys. Rev. D, 80 (2009), 076001; arXiv: 0811.4405.

[7] Л. В. Келдыш, Докт. дисс., ФИАН, 1965; E. O. Kane, Phys. Rev., 131:1 (1963), 79-88; В.Л. Бонч-Бруевич (ред.), Вопросы электронной теории силъно легированных полупроводников, Итоги науки. Физика твердого тела. Теория твердого тела, ВИНИТИ, М., 1965. 